\title{
Low energy analysis techniques for CUORE
}

C. Alduino ${ }^{1}$, K. Alfonso ${ }^{2}$, D. R. Artusa $a^{1,3}$, F. T. AvignoneIII ${ }^{1}$, O. Azzolini ${ }^{4}$, G. Bari ${ }^{5}$, J. W. Beeman ${ }^{6}$, F. Bellini ${ }^{7,8}$, G. Benato ${ }^{9}$, A. Bersani ${ }^{10}$, M. Biassoni11,12, A. Branca ${ }^{13}$, C. Brofferio ${ }^{11,12}$, C. Bucci ${ }^{3}$, A. Camacho ${ }^{4}$, A. Caminata ${ }^{10}$, L. Canonica ${ }^{3,14}$, X. G. Cao ${ }^{15}$, S. Capelli ${ }^{11,12}$, L. Cappelli ${ }^{3}$, L. Cardani ${ }^{8}$, P. Carniti ${ }^{11,12}$, N. Casali ${ }^{8}$, L. Cassina ${ }^{11,12}$, D. Chiesa ${ }^{11,12}$, N. Chott ${ }^{1}$, M. Clemenza ${ }^{11,12}$, S. Copello ${ }^{10,16}$, C. Cosmelli ${ }^{7,8}$, O. Cremonesi ${ }^{12, a}$, R. J. Creswick ${ }^{1}$, J. S. Cushman ${ }^{17}$, A. D'Addabbo ${ }^{3}$, D. D'Aguanno ${ }^{3,18}$, I. Dafinei ${ }^{8}$, C. J. Davis ${ }^{17}$, S. Dell'Oro ${ }^{3,19}$, M. M. Deninno ${ }^{5}$, S. Di Domizio ${ }^{10,16}$, M. L. Di Vacri ${ }^{3,20}$, A. Drobizhev ${ }^{9,21}$, D. Q. Fang ${ }^{15}$, M. Faverzani ${ }^{11,12}$, E. Ferri ${ }^{12}$, F. Ferroni ${ }^{7,8}$, E. Fiorini ${ }^{11,12}$, M. A. Franceschi ${ }^{22}$, S. J. Freedman ${ }^{9,21, b}$, B. K. Fujikawa ${ }^{21}$, A. Giachero ${ }^{12}$, L. Gironi ${ }^{11,12}$, A. Giuliani ${ }^{23}$, L. Gladstone ${ }^{14}$, P. Gorla ${ }^{3}$, C. Gotti ${ }^{11,12}$, T. D. Gutierrez ${ }^{24}$, E. E. Haller ${ }^{6,25}$, K. Han ${ }^{26}$, E. Hansen ${ }^{2,14}$, K. M. Heeger ${ }^{17}$, R. Hennings-Yeomans ${ }^{9,21}$, H. Z. Huang ${ }^{2}$, R. Kadel ${ }^{27}$, G. Keppel ${ }^{4}$, Yu. G. Kolomensky ${ }^{9,21}$,

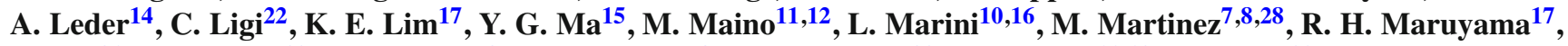
Y. Mei $^{21}$, N. Moggi ${ }^{5,29}$, S. Morganti ${ }^{8}$, P. J. Mosteiro ${ }^{8}$, T. Napolitano ${ }^{22}$, M. Nastasi ${ }^{11,12}$, C. Nones ${ }^{30}$, E. B. Norman ${ }^{31,32}$, V. Novati ${ }^{23}$, A. Nucciotti ${ }^{11,12}$, T. O'Donnell ${ }^{33}$, J. L. Ouellet ${ }^{14}$, C. E. Pagliarone ${ }^{3,18}$, M. Pallavicini ${ }^{10,16}$, V. Palmieri ${ }^{4}$, L. Pattavina ${ }^{3}$, M. Pavan ${ }^{11,12}$, G. Pessina ${ }^{12}$, G. Piperno ${ }^{7,8, c}$, C. Pira ${ }^{4}$, S. Pirro ${ }^{3}$, S. Pozzi ${ }^{11,12}$, E. Previtali ${ }^{12}$, C. Rosenfeld ${ }^{1}$, C. Rusconi ${ }^{1,3}$, M. Sakai ${ }^{2}$, S. Sangiorgio ${ }^{31}$, D. Santone ${ }^{3,20}$, B. Schmidt ${ }^{21}$, J. Schmidt ${ }^{2}$, N. D. Scielzo ${ }^{31}$, V. Singh ${ }^{9}$, M. Sisti ${ }^{11,12}$, A. R. Smith ${ }^{21}$, L. Taffarello ${ }^{13}$, F. Terranova ${ }^{11,12}$, C. Tomei ${ }^{8}$, M. Vignati ${ }^{8}$, S. L. Wagaarachchi ${ }^{9,21}$, B. S. Wang ${ }^{31,32}$, H. W. Wang ${ }^{15}$, B. Welliver ${ }^{21}$, J. Wilson ${ }^{1}$, L. A. Winslow ${ }^{14}$, T. Wise ${ }^{17,34}$, A. Woodcraft ${ }^{35}$, L. Zanotti ${ }^{11,12}$, G. Q. Zhang ${ }^{15}$, S. Zimmermann ${ }^{36}$, S. Zucchelli ${ }^{5,29}$

1 Department of Physics and Astronomy, University of South Carolina, Columbia, SC 29208, USA

2 Department of Physics and Astronomy, University of California, Los Angeles, CA 90095, USA

3 INFN-Laboratori Nazionali del Gran Sasso, Assergi, 67100 L'Aquila, Italy

4 INFN-Laboratori Nazionali di Legnaro, Legnaro, 35020 Padua, Italy

5 INFN-Sezione di Bologna, 40127 Bologna, Italy

6 Materials Science Division, Lawrence Berkeley National Laboratory, Berkeley, CA 94720, USA

Dipartimento di Fisica, Sapienza Università di Roma, 00185 Rome, Italy

8 INFN-Sezione di Roma, 00185 Rome, Italy

9 Department of Physics, University of California, Berkeley, CA 94720, USA

10 INFN-Sezione di Genova, 16146 Genoa, Italy

1 Dipartimento di Fisica, Università di Milano-Bicocca, 20126 Milan, Italy

2 INFN-Sezione di Milano Bicocca, 20126 Milan, Italy

3 INFN-Sezione di Padova, 35131 Padua, Italy

4 Massachusetts Institute of Technology, Cambridge, MA 02139, USA

5 Chinese Academy of Sciences, Shanghai Institute of Applied Physics, Shanghai 201800, China

6 Dipartimento di Fisica, Università di Genova, 16146 Genoa, Italy

7 Department of Physics, Yale University, New Haven, CT 06520, USA

18 Dipartimento di Ingegneria Civile e Meccanica, Università degli Studi di Cassino e del Lazio Meridionale, Cassino 03043, Italy

19 INFN-Gran Sasso Science Institute, 67100 L'Aquila, Italy

20 Dipartimento di Scienze Fisiche e Chimiche, Università dell'Aquila, 67100 L'Aquila, Italy

21 Nuclear Science Division, Lawrence Berkeley National Laboratory, Berkeley, CA 94720, USA

22 INFN-Laboratori Nazionali di Frascati, Frascati, 00044 Rome, Italy

23 CSNSM, Univ. Paris-Sud, CNRS/IN2P3, Université Paris-Saclay, 91405 Orsay, France

4 Physics Department, California Polytechnic State University, San Luis Obispo, CA 93407, USA

5 Department of Materials Science and Engineering, University of California, Berkeley, CA 94720, USA

26 Department of Physics and Astronomy, Shanghai Jiao Tong University, Shanghai 200240, China

7 Physics Division, Lawrence Berkeley National Laboratory, Berkeley, CA 94720, USA

8 Laboratorio de Fisica Nuclear y Astroparticulas, Universidad de Zaragoza, 50009 Saragossa, Spain

29 Dipartimento di Fisica e Astronomia, Alma Mater Studiorum-Università di Bologna, 40127 Bologna, Italy

Service de Physique des Particules, CEA/Saclay, 91191 Gif-sur-Yvette, France

1 Lawrence Livermore National Laboratory, Livermore, CA 94550, USA

2 Department of Nuclear Engineering, University of California, Berkeley, CA 94720, USA

33 Center for Neutrino Physics, Virginia Polytechnic Institute and State University, Blacksburg, VA 24061, USA

34 Department of Physics, University of Wisconsin, Madison, WI 53706, USA 
35 SUPA, Institute for Astronomy, University of Edinburgh, Blackford Hill, Edinburgh EH9 3HJ, UK

36 Engineering Division, Lawrence Berkeley National Laboratory, Berkeley, CA 94720, USA

Received: 24 August 2017 / Accepted: 30 November 2017 / Published online: 12 December 2017

(C) The Author(s) 2017. This article is an open access publication

\begin{abstract}
CUORE is a tonne-scale cryogenic detector operating at the Laboratori Nazionali del Gran Sasso (LNGS) that uses tellurium dioxide bolometers to search for neutrinoless double-beta decay of ${ }^{130} \mathrm{Te}$. CUORE is also suitable to search for low energy rare events such as solar axions or WIMP scattering, thanks to its ultra-low background and large target mass. However, to conduct such sensitive searches requires improving the energy threshold to $10 \mathrm{keV}$. In this paper, we describe the analysis techniques developed for the low energy analysis of CUORE-like detectors, using the data acquired from November 2013 to March 2015 by CUORE-0, a singletower prototype designed to validate the assembly procedure and new cleaning techniques of CUORE. We explain the energy threshold optimization, continuous monitoring of the trigger efficiency, data and event selection, and energy calibration at low energies in detail. We also present the low energy background spectrum of CUORE- 0 below $60 \mathrm{keV}$. Finally, we report the sensitivity of CUORE to WIMP annual modulation using the CUORE-0 energy threshold and background, as well as an estimate of the uncertainty on the nuclear quenching factor from nuclear recoils inCUORE-0.
\end{abstract}

\section{Introduction}

CUORE (Cryogenic Underground Observatory for Rare Events) is a tonne-scale cryogenic detector primarily designed to search for the neutrinoless double-beta $(0 \nu \beta \beta)$ decay of ${ }^{130} \mathrm{Te}[1,2]$. In $0 v \beta \beta$ decay, two neutrons in an atomic nucleus simultaneously decay to two protons and two electrons, without emitting any electron antineutrinos. The experimental signature of $0 v \beta \beta$ decay is a sharp peak at the tail end of the two-neutrino double-beta decay $(2 \nu \beta \beta)$ summed energy spectrum. The bolometric technique of CUORE offers an excellent energy resolution of $\sim 5 \mathrm{keV}$ FWHM at the $Q$ value of ${ }^{130} \mathrm{Te}, 2527.5 \mathrm{keV}$ [3-5], which suppresses the $2 \nu \beta \beta$ decay background leaking into the $0 \nu \beta \beta$ decay signal region of interest (ROI) [6].

The CUORE program builds on a predecesor experiment, Cuoricino, which reported a lower limit on the ${ }^{130} \mathrm{Te} 0 \nu \beta \beta$ decay half-life of $2.8 \times 10^{24}$ year $(90 \%$ C.L.) with data

a e-mail: cuore-spokesperson@lngs.infn.it

b Deceased

c Presently at: INFN-Laboratori Nazionali di Frascati, Frascati, 00044 Rome, Italy accumulated from 2003 to 2008 [7]. The successor experiment CUORE-0, operated from 2013 to 2015, set a limit of $4.0 \times 10^{24}$ year $(90 \%$ C.L.) in combination with the Cuoricino data [8]. CUORE is currently in data-taking at LNGS.

While CUORE will be one of the leading $0 \nu \beta \beta$ decay experiments during its scheduled 5 years of data-taking, it will also benefit from the ultra-low background and large target mass to search for lower energy rare events, such as the direct detection of Weakly Interacting Massive Particle (WIMP) dark matter or solar axions [9]. WIMP direct detection is possible with terrestrial detectors by measuring nuclear recoils produced as WIMPs scatter off nuclei in the target material [10]. The resulting energy spectrum falls quasi-exponentially as a function of energy and extends to only a few tens of $\mathrm{keV}$ for typical WIMPs with masses of $\mathcal{O}\left(100 \mathrm{GeV} / \mathrm{c}^{2}\right)$. For WIMPs in the galactic halo, an annual modulation of the event rate is expected due to the Earth's motion relative to the dark matter halo of the Milky Way [11,12], with event rates highest in June, when the Earth's relative velocity with respect to the halo is maximal, and lowest in December. Alternatively, solar axions can be detected by the inverse Primakoff effect in the Coulomb field of the crystal, with a signal from the M1 transition of ${ }^{57} \mathrm{Fe}$ expected at $14.4 \mathrm{keV}$ [13]. A critical requirement for both these searches is the achievement of an energy threshold of $<10 \mathrm{keV}$ and sufficient rejection of low energy noise and/or spurious events, in addition to a detailed understanding of the low energy backgrounds and adequate detector stability.

Since Cuoricino, which proved that the CUORE detector technology is well suited for searching for $0 \nu \beta \beta$ decay [7], the CUORE collaboration has worked to lower the energy thresholds to perform low energy rare event searches. We developed a new low energy software trigger, the "optimal trigger" (OT) [14], based on filtering the continuous data stream before the application of the trigger condition. We implemented this algorithm in test measurements of the bolometric performance of a small number of CUORE crystals in a dedicated setup (CUORE Crystal Validation Runs, CCVR) [15]. The results were encouraging; we were able to identify events with energies as low as $3 \mathrm{keV}$ with a trigger efficiency of $>90 \%$ in three bolometers out of four [16]. In CUORE-0, we improved the OT technique and developed new software and hardware tools for the low energy analysis. These tools include the continuous monitoring of the trigger efficiency, the development of low energy event selection criteria, and a low energy calibration. 
In this paper, we describe our low energy analysis techniques in detail, present the energy thresholds and spectrum from the CUORE-0 experiment and report the sensitivity of CUORE to WIMP-induced annual modulation assuming the same energy thresholds and background. We also evaluate the uncertainty on the nuclear quenching factor of $\mathrm{TeO}_{2}$ obtained from CUORE-0 data. Specifically, Sect. 2 describes the experimental setup and data production of CUORE-0. Section 3 explains the OT algorithm and trigger efficiency evaluation, and Sect. 4 details the data and event selection criteria. Section 5 presents the low energy spectrum as well as the determination of the analysis threshold and the evaluation of the low energy calibration uncertainty. Section 6 outlines the analysis developed for a WIMP search, including the nuclear quenching factor of $\mathrm{TeO}_{2}$ estimation, as well as a study of the sensitivity of CUORE to WIMP-induced annual modulation. Finally, we present the summary in Sect. 7.

\section{The CUORE-0 experiment}

CUORE-0 comprised $52 \mathrm{TeO}_{2}$ crystals with a total active mass of $38.4 \mathrm{~kg}$. The crystals were arranged in a single tower, with 13 planes of four $5 \times 5 \times 5 \mathrm{~cm}^{3}$ crystals held securely inside a copper frame by polytetrafluoroethylene supports. The detector was hosted in the same cryostat used for Cuoricino at a base temperature of $\sim 10 \mathrm{mK}$, and used the same shielding and electronics. The detector design, construction, and operation are detailed in [17]. Each crystal was instrumented with a neutron-transmutation-doped (NTD) germanium thermistor [18] to read the thermal signal, and a silicon resistor [19], used as a Joule heater to inject reference pulses of constant energy every $300 \mathrm{~s}$. The reference pulses were mainly used to correct the thermal gain against the drift in temperature, but they also played a fundamental role in determining the OT efficiency, as explained in Sect. 3. The thermal readout of each bolometer thermistor was in the form of a voltage waveform continuously acquired with a sampling frequency of $125 \mathrm{~S} / \mathrm{s}$. Taking advantage of this relatively low acquisition rate, we could record the continuous data stream without hardware trigger. This allowed us to reprocess the raw data with different software trigger algorithms and to optimize the energy thresholds offline.

We collected data in one-day-long runs. Approximately once per month, we calibrated the detector by irradiating it for about 3 days using thoriated tungsten wires inserted between the outer vacuum chamber of the cryostat and the external lead shielding. The basic analysis unit is the dataset, which is composed of initial calibration runs, approximately 3 weeks of physics runs, and final calibration runs. For the low energy analysis, we also performed a dedicated measurement before each final calibration using low energy pulses generated by the Joule heater, with energies ranging from 0 to $50 \mathrm{keV}$.
A comprehensive description of the standard CUORE- 0 data processing procedure for $0 \nu \beta \beta$ decay and $2 \nu \beta \beta$ decay can be found in [20]. The following summarizes the major steps of the data processing that are common to both low energy and high energy ( $0 \nu \beta \beta$ decay and $2 \nu \beta \beta$ decay) analyses. After application of the software trigger, we store events in $5 \mathrm{~s}$ windows and evaluate the pulse amplitude using the optimal filter (OF) [21]. The OF weights each frequency component by the expected signal-to-noise ratio, calculated as the ratio between the average pulse (obtained from the $2615 \mathrm{keV} \gamma$ rays in the calibration data), and the average noise power spectra (NPS). To calculate the NPS we average baselines recorded in windows without a signal, that are acquired simultaneously on all bolometers every $200 \mathrm{~s}$ and selected for the NPS after some quality checks (basically we require that no pulses or pulse tails are present in the window). The drift in signal gain due to temperature fluctuations in the bolometer is corrected by performing a linear regression between the detector baseline voltage, a proxy for the detector temperature, and the amplitude of the reference pulser events. Finally, the voltage readout is converted to energy using the numerous $\gamma$ ray peaks between 511 and $2615 \mathrm{keV}$ from the daughter nuclei of ${ }^{232} \mathrm{Th}$ in the calibration data. The mapping from pulse amplitude to energy is described as a second-order polynomial with zero intercept to take into account possible nonlinearities, as those originated from the pulse shape dependence on energy.

\section{Optimal trigger optimization and efficiency evaluation}

The energy ROI of the standard data processing for $0 v \beta \beta$ decay analysis is in the $\mathrm{MeV}$ range, and we use a simple trigger algorithm which flags an event when the slope of the waveform exceeds a given threshold for a certain amount of time [17]. This results in energy thresholds ranging from 30 to $120 \mathrm{keV}$, depending on the bolometer, while the use of the OT algorithm is critical for lowering the threshold below $30 \mathrm{keV}$.

The OT algorithm works as follows. The data buffer is divided into slices that are continuously filtered in the frequency domain with the OF described in Sect. 2. The filtered waveforms are less noisy than the original waveforms, and baseline fluctuations are reduced. This allows us to trigger on the filtered trace in the time domain with a simple threshold as low as $<10 \mathrm{keV}$. Furthermore, the filter is sensitive to the shape of the expected signal, suppressing trigger on spurious noise-induced pulses.

The algorithm used in CUORE-0 is improved relative to that described in [14] in order to achieve a higher trigger efficiency. In particular, we have removed the veto around high energy pulses that prevented the algorithm from re-triggering 


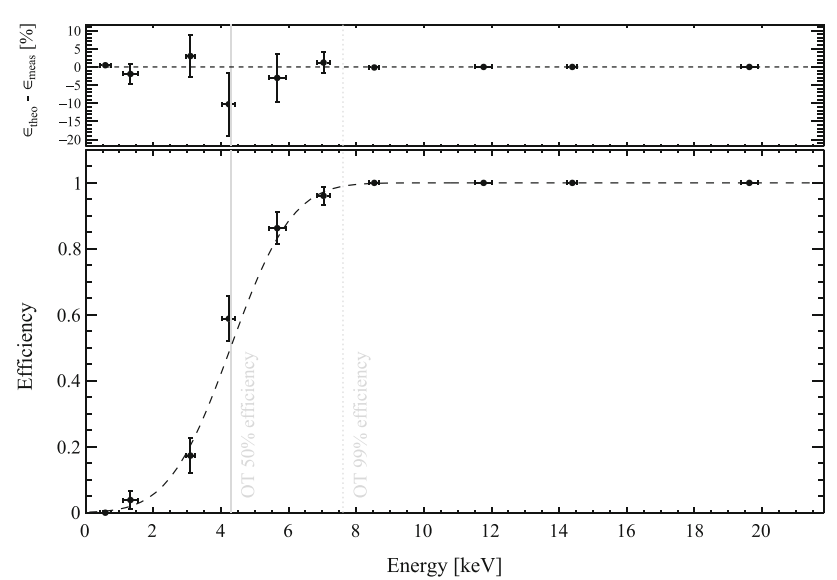

Fig. 1 (Bottom): An example of the trigger efficiency obtained from Eq. 1 as a function of energy (dashed line) and data obtained from low energy pulser measurements (circles). Energies where OT trigger efficiency reaches $50 \%(\theta)$ and $99 \%\left(E_{99 \%}\right.$ trig $)$ are shown as vertical gray lines. (Top): The difference between the model and the data

the symmetric side lobes generated by the OF, which was identified in [14] as the main source of trigger inefficiency. The new algorithm recognizes the side lobes of a high energy pulse as OF artifacts and does not flag them.

We set the trigger threshold independently for every bolometer in every dataset (hereafter "BoDs") based on the noise level. First, we calculate an OT trigger level at $\theta=3 \sigma_{\mathrm{OF}}$, where $\sigma_{\mathrm{OF}}$ is the baseline resolution after applying the $\mathrm{OF}$. The energy-dependent trigger efficiency $\varepsilon(E)$ is modeled by the Gaussian cumulative density function

$\varepsilon(E)=\frac{1}{2} \operatorname{erf}\left(\frac{E-\theta}{\sqrt{2} \sigma_{\mathrm{OF}}}\right)+\frac{1}{2}$,

which is $50 \%$ for $E=\theta$. At this energy we reject $99.86 \%$ of baseline noise. The trigger threshold, $E_{99 \%}$ trig, is set to the value at which $99 \%$ efficiency is reached. The validity of the efficiency calculation is checked at the end of each dataset with dedicated measurements injecting low energy pulses [22].

The bottom plot of Fig. 1 shows an example of trigger efficiency (dashed line) as a function of energy, along with data obtained from corresponding low energy pulser measurements (circles). Vertical gray lines indicate the energies where OT trigger efficiency reaches 50\% $(\theta)$ and $99 \%$ $\left(E_{99 \% \text { trig }}\right.$ ). In this case, $E_{99 \%}$ trig threshold is set at $7.5 \mathrm{keV}$. The top plot shows the difference between the modeled trig- ger efficiency and the data. The CUORE-0 $E_{99 \%}$ trig thresholds range from 4 to $12 \mathrm{keV}$ for most BoDs, slightly above those of the CCVR measurement due to a larger noise contribution, as explained in Sect. 4.3. In Table 1 we summarize the different energy thresholds considered in this work together with the range of values obtained for the CUORE-0 BoDs.

\section{Data selection and energy threshold determination}

In this section we detail three steps to select legitimate low energy events. First, we only choose data whose low energy response and stability are verified. Second, we identify and remove non-legitimate events that pass the trigger requirement, such as electronic noise, tower vibrations, pile-ups or particle interactions in a thermistor, where the last appears as a narrow pulse with fast decay time. Last, we remove events that occur simultaneously in more than one bolometer since the probability that WIMP or solar axion interactions occur in more than one bolometer within the coincidence window is essentially zero. In this way we reject muons passing through the tower and radioactive decays that deposit energy in several bolometers ( $\alpha$-decays in the surfaces of the crystals, Compton scattering, cascade $\gamma$-rays...).

\subsection{Dataset-bolometer selection criteria}

While CUORE-0 ran from March 2013 to March 2015, we only use 11 datasets from the second data-taking campaign, lasting from November 2013 to March 2015, because of its more stable cryogenic conditions. We exclude some runs (a total of $\sim 5$ data-taking days) with an abnormally higher ( $\sim$ 10 times) low energy event rate, which we attribute to cryostat instability following a helium refill. To preserve the data quality, we reject the time intervals for each bolometer that exhibit degraded bolometric performance due to large baseline excursions or elevated noise levels, as described in [20]. The total exposure after these preliminary quality checks is $23.15 \mathrm{~kg}$ year of $\mathrm{TeO}_{2}$.

To ensure a stable energy calibration and sufficient resolution at low energies, we additionally require that the residual gain variation of the energy pulser of every BoDs after temperature stabilization do not vary more than $\pm\left(\sigma_{\mathrm{DS}}+1 \mathrm{keV}\right)$ from the mean over the entire data-taking period, where $\sigma_{\mathrm{DS}}$
Table 1 Different energy thresholds considered in this work (see Sects. 3 and 4). The last column represent the range of values obtained for the CUORE-0 BoDs

\begin{tabular}{llll}
\hline Symbol & Name & Description & Energy range (keV) \\
\hline$\theta$ & OT trigger level & Trigger firing energy & $2-7$ \\
$E_{99 \% \text { trig }}$ & Trigger threshold & Energy for 99\% trigger efficiency & $4-12$ \\
$E_{\text {thres }}$ & Energy threshold & Lower noise-free energy & $8-35$ \\
\hline
\end{tabular}




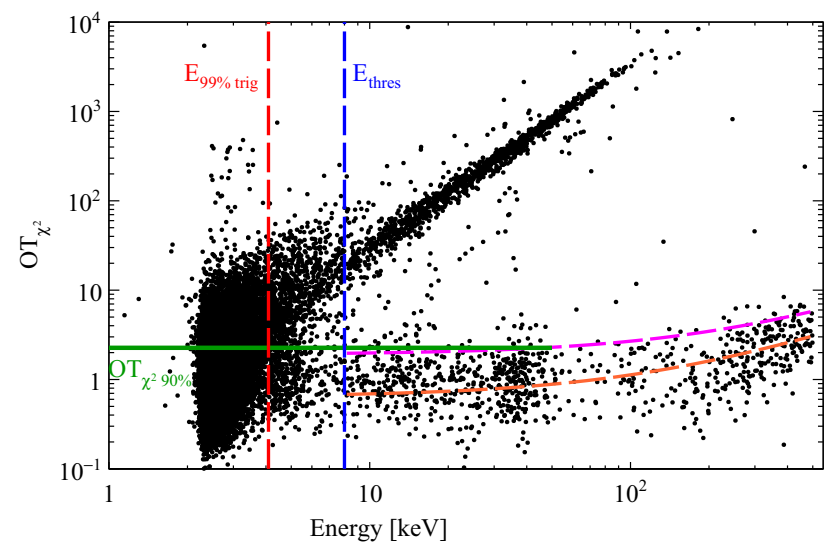

Fig. 2 A typical distribution of $O T_{\chi^{2}}$ as a function of energy for one BoDs. Physical events due to the particle interactions in the $\mathrm{TeO}_{2}$ crystals are distributed in a nearly-horizontal band around $O T_{\chi^{2}} \sim 1$. Nonphysical events such as electronic noise and tower vibrations, as well as particle interactions in the thermistors, follow an oblique distribution. The green solid line corresponds to the 90th percentile of the $O T_{\chi^{2}}$ distribution $\left(O T_{\chi^{2} 90 \%}\right)$ calculated in the region [35-50] keV and $O T_{\chi^{2}}<10$ using physics data. The magenta (orange) dashed line corresponds to the 90th (50th) percentile calculated using calibration data in the region [100-500] keV and $O T_{\chi^{2}}<10$, assuming linear dependence on energy. Red and blue dashed vertical lines represent the trigger threshold $E_{99 \%}$ trig and the analysis threshold $E_{\text {thres }}$, respectively

is the uncertainty in the pulser position associated with the dataset and the pulser energy ranges from 13 to $27 \mathrm{keV}$. We also discard BoDs with fewer than 11 events in the region where we evaluate the pulse shape parameter event selection efficiency (35-50 keV, see Sect. 4.2). Finally, we exclude run-bolometer pairs with baseline RMS values that are greater than $2 \sigma$ above the median, where median and $\sigma$ are calculated for each bolometer over all the datasets. After all the data selection, we use 490 BoDs out of 539 with a total $\mathrm{TeO}_{2}$ exposure of $20.02 \mathrm{~kg}$ year.

\subsection{Event selection criteria}

For the standard $0 \nu \beta \beta$ decay analysis we use a set of six pulse shape parameters to select physical events in $\mathrm{TeO}_{2}$ based on the pulse shape characteristics [20]. These parameters, however, lose rejection power at low energy due to the worse signal-to-noise ratio. Instead, the OT algorithm provides us with a powerful shape parameter, $O T_{\chi^{2}}$, to select legitimate signal candidate events at low energy [14]. We define $O T_{\chi^{2}}$ as the reduced $\chi^{2}$ computed between the triggered event and a cubic spline of the filtered average pulse obtained from the calibration $\gamma$ rays at $2615 \mathrm{keV}$. This parameter is sensitive to the shape of the expected signal, suppressing pulses with shape deviating from the nominal one.

Figure 2 shows a typical $O T_{\chi^{2}}$ distribution as a function of energy for the triggered events which pass the selection criteria described in Sect. 4.1. Between the OT trigger level and
$E_{99 \%}$ trig there can be a leakage of baseline noise. Physical events due to particle interactions in the $\mathrm{TeO}_{2}$ crystals scatter around $O T_{\chi^{2}} \sim 1$ forming an almost horizontal distribution, while spurious events due to electronic noise or particle interactions in the thermistors follow an oblique distribution with $O T_{\chi^{2}}$ values as high as $\sim 10^{4}$ at $200 \mathrm{keV}$. Pile-up events lie between the two bands. We select legitimate physical events with a requirement on the $O T_{\chi^{2}}$ parameter, and we evaluate the selection efficiency by counting the number of events before and after the cut in a region free of noise. As is evident in Fig. 2, $O T_{\chi}{ }^{2}$ has a slight dependence on energy; this dependence is more or less pronounced depending on the bolometer, but mostly is imperceptible below $100 \mathrm{keV}$. Assuming no energy dependence at low energy in the range between 10 and $50 \mathrm{keV}$, we compute the selection efficiency in the region with $O T_{\chi^{2}}<10$ and energy in the range $35-50 \mathrm{keV}$, where the statistics are higher and the noise leakage is negligible. We choose the values of the selection to achieve $90 \%$ efficiency and calculate it as the 90th percentile of the $O T_{\chi^{2}}$ distribution ( $O T_{\chi^{2} 90 \%}$, green solid line in Fig. 2). The selection efficiency is computed independently for every BoDs, and the uncertainty, evaluated taking into account the statistical fluctuation in counting for each BoDs, ranges from 0.3 to $1 \%$. We have investigated the $O T_{\chi^{2}}$ dependence on energy using calibration data, as the low statistics above $60 \mathrm{keV}$ make the results significantly uncertain in background data. The behaviour is well described by a linear fit up to $500 \mathrm{keV}$ and the $O T_{\chi^{2} 90 \%}$ value at $35-50 \mathrm{keV}$ agrees with that calculated in background data for bolometers featuring low rate during calibration, like the one on Fig. 2, where the magenta dashed line corresponds to $90 \%$ efficiency and the orange dashed line to $50 \%$ efficiency. However, in general the larger pileup probability during calibration runs shifts the $O T_{\chi^{2}} 90 \%$ selection upwards with respect to the value in background runs, so in the following we use the value calculated in background and assume no energy dependence down to threshold. In order to estimate the uncertainty related to the choice of an energy independent cut efficiency, we have calculated the counting rate below $35 \mathrm{keV}$ after the cut assuming the same energy dependence measured in calibration, being the difference with respect to the energy-independent selection lower than the statistical error.

The validity of the selection efficiency computation relies on the assumption that the region $35-50 \mathrm{keV}$ is free of noise and the shape of the $O T_{\chi^{2}}$ distribution does not change at lower energies. To verify this hypothesis, we compare the $O T_{\chi^{2} 90 \%}$ selection with the 50th percentile selection $\left(O T_{\chi^{2} 50 \%}\right)$, assuming their selection efficiencies are 90 and $50 \%$, respectively. If there exists a significant noise contribution with the $O T_{\chi^{2} 90 \%}$ selection, the efficiency-corrected spectra would differ, as noise rejection is stronger for the $O T_{\chi^{2} 50 \%}$ selection. The residual spectrum is shown in red in Fig. 3. The selection efficiency cor- 


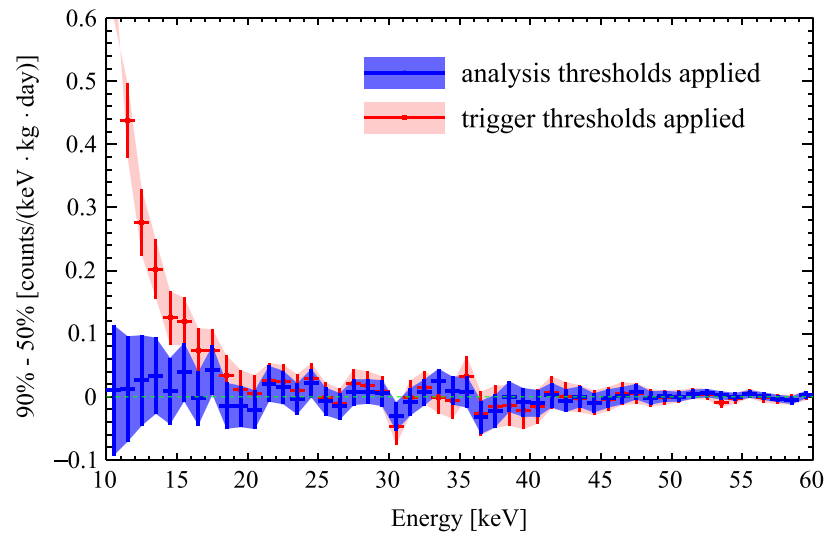

Fig. $3 O T_{\chi^{2}}$ selection efficiency corrected rate difference between the background spectra calculated for $O T_{\chi^{2} 90 \%}$ and $O T_{\chi^{2} 50 \%}$. (See Sect. 4.3 for a description of analysis and trigger thresholds.)

rected rate difference between the two spectra is compatible with zero down to $\sim 25 \mathrm{keV}$. Below $\sim 25 \mathrm{keV}$ the rate difference increases, suggesting the presence of noise in the data. In fact, the noise contribution for most bolometers overlaps the physical-events band at energies directly

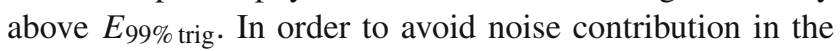
spectrum we set the most stringent analysis energy thresh-

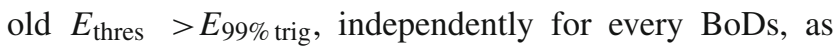
described in Sect. 4.3.

\subsection{Energy threshold determination}

The CUORE-0 cryostat at LNGS was more noisy than the R\&D cryostat in which the CCVR bolometer performance tests were performed. It means that in CUORE-0,

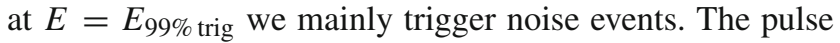
shape parameter $O T_{\chi^{2}}$ presented in Sect. 4.2 provides powerful discrimination between physics and spurious events, but the two populations overlap as the energy decreases.

In order to avoid a leakage of spurious events in the data we set an analysis threshold ( $\left.E_{\text {thres }}\right)$ at the minimum energy where the populations are well separated for each BoDs. Specifically, we perform a Kolmogorov-Smirnov (KS) test to quantify the similarity between the $O T_{\chi^{2}}$ populations in different energy slices with respect to the $O T_{\chi^{2}}$ distribution at $35-50 \mathrm{keV}$ and $O T_{\chi^{2}}<10$, the same pure signal sample region used to calculate the $O T_{\chi^{2}}$ selection efficiency. Start-

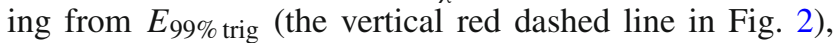
we compare the distribution of $O T_{\chi^{2}}$ in $4 \mathrm{keV}$-width energy windows with the reference distribution, for $O T_{\chi^{2}}<10$. We set $E_{\text {thres }}$ at the lower edge of the energy range that provides $\mathrm{KS}$ probability larger than 0.1 . This method is not valid when the reference sample is contaminated with noise. We ensure the validity of the reference by requiring $O T_{\chi^{2}} 90 \%<6$. The value 6 is obtained from the $O T_{\chi^{2} 90 \%}$ distribution of all BoDs as the $2 \sigma$ above the median. We discard 37 BoDs that do not fulfill this requirement from a total of 490. Once the KS threshold was fixed, the technique described worked for all 490 BoDs without manual adjustments, making it suitable for an $\mathcal{O}(1000)$ bolometers experiment.

Final analysis thresholds range from 8 to $35 \mathrm{keV}$, with only 16 BoDs having a threshold lower than $10 \mathrm{keV}$, thus not being representative of the whole data-taking. Therefore, we set $10 \mathrm{keV}$ as the minimum CUORE-0 energy threshold. The exposure ranges from $1.6 \mathrm{~kg}$ year at $10 \mathrm{keV}$ up to $18.56 \mathrm{~kg}$ year at $35 \mathrm{keV}$ (see inset of Fig. 5). We verify that the noise acceptance is negligible with the same procedure used in Sect. 4.2. As shown as the blue band in Fig. 3, the 90-50\% residual with the analysis thresholds is compatible with zero down to $10 \mathrm{keV}$.

\subsection{Anti-coincidence requirement}

The last event selection criterion for the low energy rare event searches is anti-coincidence; i.e., no signal events are triggered in other bolometers in a certain temporal window. We use a coincidence window of $\pm 100 \mathrm{~ms}, 20$ times wider than that used for the standard $0 \nu \beta \beta$ decay analysis [20], due to the larger difference in characteristic rise time between low and high energy events. To evaluate the event loss due to random coincidences between physical events and unrelated events on another bolometer (anticoincidence selection efficiency) we use the $1461 \mathrm{keV} \gamma$ ray peak in the single crystal energy spectrum. This peak, coming from ${ }^{40} \mathrm{~K} \mathrm{EC}$, does not belong to any cascade, so the only true coincident event is the $\sim 3 \mathrm{keV}$ X-ray from the Ar de-excitation, which is below our threshold. Counting the number of events in the $1461 \mathrm{keV}$ peak of the single crystal spectrum before and after the selection we find the anti-coincidence selection efficiency to be $99.2 \pm 0.3 \%$. We combine this efficiency with the $90 \%$ event selection efficiency on $O T_{\chi^{2}}$ to obtain the total detection efficiency. The uncertainty on the efficiency is BoDs dependent.

\section{Low energy spectrum construction}

\subsection{Energy calibration}

During the calibration runs, the ${ }^{232} \mathrm{Th}$ sources are outside the cryostat, so the $\gamma$ rays pass through a 1.4-cm-thick ancient Roman lead shield before reaching the detector. Consequently, the peaks in the low energy region of the spectrum are highly attenuated, and only those between 511 and $2615 \mathrm{keV}$ are clearly visible and used to calibrate the energy response of each bolometer. As stated in Sect. 2, in the standard $0 \nu \beta \beta$ decay analysis we use a second-order polynomial with zero intercept to fit the reconstructed peak positions in the calibration spectrum to their nominal energies. To improve the energy resolution at the $Q$-value of $0 \nu \beta \beta$ 
Table 2 Main Te X-ray emission lines. Data from [23]

\begin{tabular}{lll}
\hline Line & Energy $(\mathrm{keV})$ & Intensity $(\%)$ \\
\hline $\mathrm{K} \alpha 1$ & 27.472 & 47.1 \\
$\mathrm{~K} \alpha 2$ & 27.202 & 25.3 \\
$\mathrm{~K} \alpha 3$ & 26.875 & 0.00202 \\
$\mathrm{~K} \beta 1$ & 30.995 & 8.19 \\
$\mathrm{~K} \beta 2$ & 31.704 & 2.37 \\
$\mathrm{~K} \beta 3$ & 30.944 & 4.25 \\
$\mathrm{~K} \beta 4$ & 31.774 & 0.363 \\
$\mathrm{~K} \beta 5$ & 31.237 & 0.075 \\
\hline
\end{tabular}

decay, a combination of four energy estimators with slightly different calibration coefficients are used in the final analysis presented in [8]. For the low energy analysis, we use a single energy estimator to avoid complexity.

The calibration uncertainty in the $0 v \beta \beta$ decay ROI is $0.05 \pm 0.05$ (stat.) \pm 0.09 (syst.) $\mathrm{keV}$ [20], but this value is energy dependent. To validate the extrapolation to energies below $100 \mathrm{keV}$, we use the characteristic Te X-rays that can follow a $\gamma$ ray interaction, which can escape the crystal and be detected in another crystal. These events can be selected by requiring a coincidence in an adjacent crystal. The most intense $\mathrm{X}$-rays are eight $\mathrm{K}$-shell peaks ranging from 26 to $32 \mathrm{keV}$ (see Table 2). Due to the energy resolution, these peaks are noticeable as a main and a secondary peak around 27 and $31 \mathrm{keV}$, in both the calibration and background spectra.

To determine their reconstructed energies, we fit the region from 22 to $34 \mathrm{keV}$ with an eight-Gaussian line shape plus a linear background, where all of the Gaussians are constrained to have the same width. The relative intensities and positions of each Gaussian are fixed with respect to the main $\mathrm{K}_{\alpha 1}$ peak using nuclear data from [23]. In order to take into account any possible discrepancy in the relative intensities of the peaks arising from systematic effects in the detector, we determine these intensities with a Monte Carlo (MC) simulation based on the Geant4 package [24] (version 4.9.6.p03, see [25] for details) that includes the bolometer-dependent energy thresholds and the analysis coincidence window.

Figure 4 displays the fit results of both calibration and physics data. The most intense $\mathrm{K}_{\alpha 1}$ peaks for calibration and physics data are measured to be $27.60 \pm 0.05 \mathrm{keV}$ and $27.65 \pm 0.13 \mathrm{keV}$, respectively. The corresponding residuals with respect to the nominal energy are $0.13 \pm 0.05 \mathrm{keV}$ and $0.18 \pm 0.13 \mathrm{keV}$, respectively. The latter indicates the systematic upward shift on the energy scale in the physics data, and we take into account its impact on the WIMP sensitivity reported in Sect. 6.

The difference in peak positions between the nuclear data and the simulation is found to be less than $0.01 \mathrm{keV}$. Never-
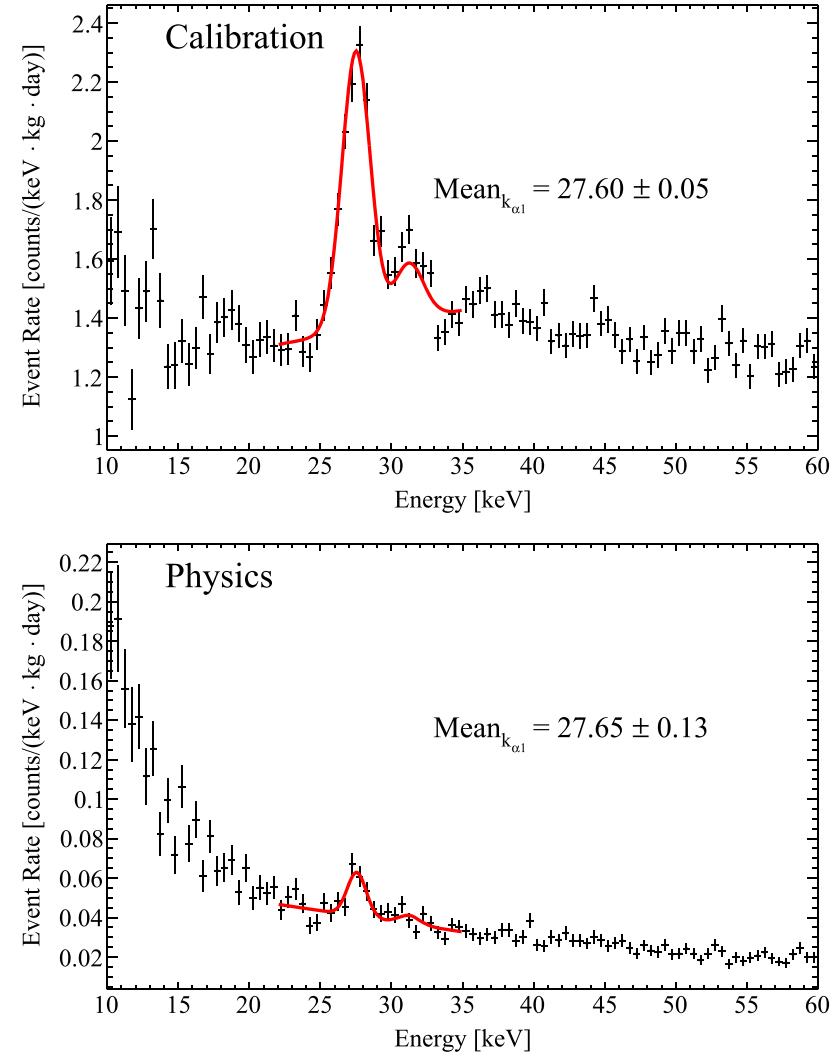

Fig. 4 CUORE-0 summed energy spectra of events with doublecrystal coincidence in calibration (top) and physics (bottom) data, along with fits to an eight-Gaussian line shape plus linear background (red solid lines)

theless, the amplitude ratio $\mathrm{K}_{\beta 1} / \mathrm{K}_{\alpha 1}$ in the $\mathrm{MC}$ simulation is 0.27 instead of 0.17 from Table 2 due to the strong change in $\mathrm{X}$-ray attenuation length between 27 and $31 \mathrm{keV}$. This effect is also appreciable in the CUORE-0 data; as shown in Fig. 4 (upper panel) the $31 \mathrm{keV}$ peak is underestimated by the fit function. Leaving the relative intensity $\mathrm{K}_{\beta 1} / \mathrm{K}_{\alpha 1}$ as a floating parameter improves the goodness of the fit and reproduces the relative intensities estimated by MC, but the position of the $\mathrm{K}_{\alpha 1}$ peak does not change within the uncertainty.

\subsection{Energy spectrum}

Figure 5 shows the low energy spectrum of CUORE-0, using the selected BoDs with the event selection criteria described in Sect. 4 and the detection efficiency. The background rate above $50 \mathrm{keV}$ is 0.05 counts / $(\mathrm{kg} \mathrm{keV}$ day), consistent with the results obtained in the standard $0 \nu \beta \beta$ decay analysis. Below $50 \mathrm{keV}$, the background rate increases substantially to 1.7 counts/ ( $\mathrm{kg} \mathrm{keV}$ day) at $10 \mathrm{keV}$; it is, however, two times lower than the background rate measured with the four best Cuoricino bolometers for which thresholds below $10 \mathrm{keV}$ were attained [16]. 


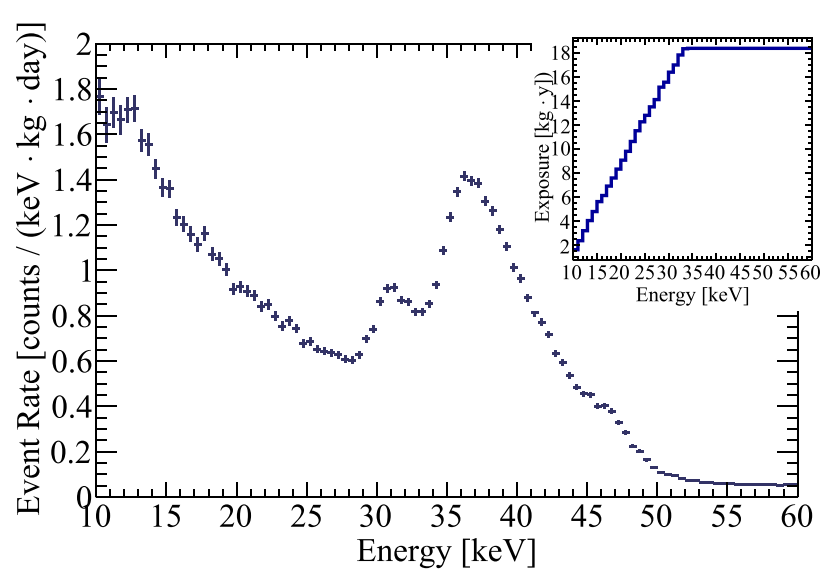

Fig. 5 Efficiency-corrected energy spectrum of CUORE-0 from 10 to $60 \mathrm{keV}$ corresponding to an exposure ranging from $1.6 \mathrm{~kg}$ year at $10 \mathrm{keV}$ up to $18.56 \mathrm{~kg}$ year at $35 \mathrm{keV}$, as shown in the inset

The most noticeable feature in the spectrum is a peaklike structure around 31 and $37 \mathrm{keV}$. Given that this structure is observed in all the bolometers and it was also present in the Cuoricino background, its origin is likely physical. The current background prediction based on our MC simulation [25] does not fully account for the low energy spectrum including this peak-like structure, and further investigation is on-going under the hypothesis that the contamination is due to materials facing the detectors (e.g. copper shielding...). We expect to have better insights on this peak-like structure with CUORE data, where inner bolometers mostly face other bolometers and not the copper shielding. Through the comparison between the innermost and outermost bolometers, we may be able to attribute the origin of this peak-like structure to a certain process.

\section{CUORE sensitivity to WIMP annual modulation}

In this section we present the sensitivity of CUORE to the annual modulation in the detection rate induced by dark matter in the galactic halo. We restrict our analysis to WIMPs interacting with the target nuclei in the detector via elastic scattering off nuclei; for this reason, we present a study of the nuclear quenching factor of $\mathrm{TeO}_{2}$ obtained in CUORE-0.

\section{1 $\mathrm{TeO}_{2}$ nuclear quenching factor}

One of the prerequisites to perform a WIMP dark matter search is a good understanding on the low energy response of the detector for both nuclear recoils (NRs), produced by WIMPs or background neutrons, and electronic recoils (ERs), produced by electromagnetic backgrounds. The nuclear quenching factor is defined as the ratio of the measured signal generated by a NR to that generated by an
Table 3 Expected energy, measured energy obtained from fits, and resulting quenching factors $(\mathrm{QFs})$ for the selected recoiling nuclei. Only statistical uncertainties are shown in the QFs

\begin{tabular}{llcl}
\hline Recoiling Nuclei & $E_{\text {expected }}$ & $E_{\text {measured }}$ & QF \\
\hline${ }^{206} \mathrm{~Pb}$ & 103.12 & $95.62 \pm 0.24$ & $0.927 \pm 0.002$ (stat.) \\
${ }^{218} \mathrm{Po}$ & 100.8 & $100.0 \pm 0.9$ & $0.992 \pm 0.009$ (stat.) \\
${ }^{220} \mathrm{Rn}$ & 103.50 & $100.45 \pm 1.21$ & $0.971 \pm 0.012$ (stat.) \\
${ }^{214} \mathrm{~Pb}$ & 112.13 & $110.92 \pm 0.96$ & $0.989 \pm 0.009$ (stat.) \\
\hline
\end{tabular}

ER depositing the same energy in the detector, and depends on the energy and recoiling nucleus. Given that any energy conversion in the $\mathrm{TeO}_{2}$ bolometers finally produces signal through phonons, the nuclear quenching factor in the bolometers is expected to be close to one. The nuclear quenching factors of several recoiling nuclei in $\mathrm{TeO}_{2}$ have been measured previously using the daughter nuclei of the $\alpha$ decays from ${ }^{224} \mathrm{Ra},{ }^{220} \mathrm{Rn},{ }^{216} \mathrm{Po},{ }^{212} \mathrm{Po}$, and ${ }^{212} \mathrm{Bi}$ in the energy range between 100 and $170 \mathrm{keV}$. The result was found to be $1.025 \pm 0.01$ (stat) \pm 0.02 (syst) [26]. We exploit the same technique and estimate the nuclear quenching factors using the daughter nuclei of some $\alpha$ emitters at energies around $100 \mathrm{keV}$.

Specifically, we measure the recoiling energy of the daughter nuclei following $\alpha$ decays of ${ }^{210} \mathrm{Po},{ }^{222} \mathrm{Rn},{ }^{224} \mathrm{Ra}$, and ${ }^{218}$ Po present in the CUORE-0 crystal surfaces where either the $\alpha$ particle or the daughter nucleus escapes and is detected in an adjacent crystal. We tag these events by requiring coincidence in two crystals with a total energy within some tens of $\mathrm{keV}$ of the $Q$-value of the decay. Then we fit the spectrum of the recoiling nuclei with an asymmetrical Gaussian function with a smooth power-law tail relative to the mean to obtain the peak position. Table 3 summarizes the obtained nuclear quenching factors for the selected recoiling nuclei. While the nuclear quenching factor obtained from ${ }^{218} \mathrm{Po}\left({ }^{214} \mathrm{~Pb}\right.$ and $\left.{ }^{220} \mathrm{Rn}\right)$ is close to unity, we notice that the one obtained from ${ }^{206} \mathrm{~Pb}$ exhibits significant deviation from unity.

Acknowledging that these recoiling nuclei are surface events and energy losses might happen at the surface, we use unity as the nuclear quenching factor to set the energy scale of WIMPs in the following analysis, and conservatively estimate an uncertainty of nuclear quenching factor as $7 \%$ using the largest deviation from unity observed by ${ }^{206} \mathrm{~Pb}$. Its impact is integrated in Fig. 6 to report the WIMP sensitivity of CUORE.

\subsection{WIMP sensitivity of CUORE}

The sensitivity of CUORE-0 to annual modulation of WIMPs is limited by its relatively small exposure. However, we can use results of CUORE-0 to estimate the CUORE sensitiv- 


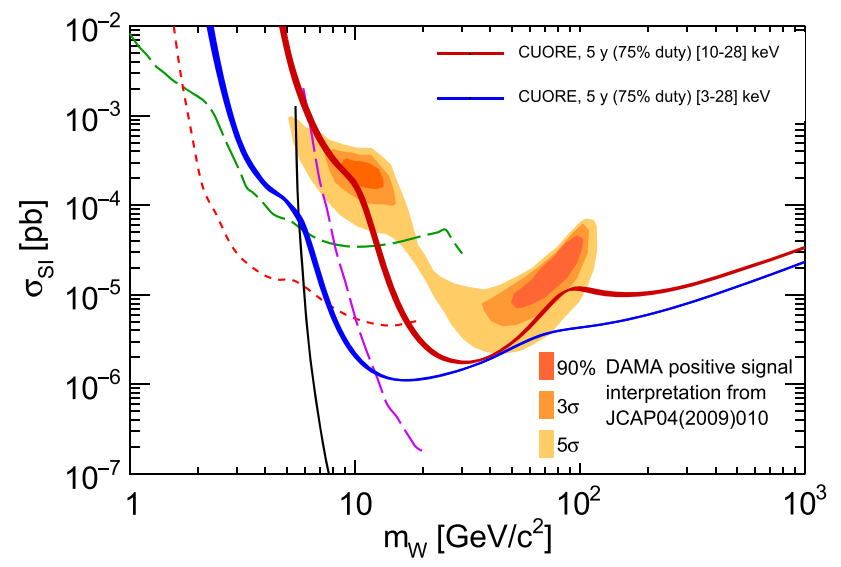

Fig. $690 \%$ sensitivities on the SI elastic WIMP-nucleon cross section as a function of WIMP mass of CUORE, assuming 5 years of data-taking with $75 \%$ of duty cycle and $10 \mathrm{keV}$ threshold (red), as well as $3 \mathrm{keV}$ threshold (blue). Uncertainty on the energy scale dominated by the nuclear quenching factor is taken into account. DAMA/LIBRA positive signal reported in [31] is shown as yellow/dark yellow/orange islands. The results from CRESST-II (dashed green) [32], CDMS Lite (dashed red) [33], XMASS (dashed violet) [34], and LUX (black solid) [35] are also shown

ity assuming the same background rate and energy thresholds. This is a conservative hypothesis since a significant background reduction is expected to be achieved in CUORE thanks to its close-packed detector configuration and the careful selection of radio-pure detector materials [25].

$\mathrm{TeO}_{2}$ is an interesting DM target, as it combines a heavy nucleus (tellurium), which provides a large scattering amplitude (assuming coherent interaction, that scales as $\mathrm{A}^{2}$ ) and a light one (oxygen) to enhance sensitivity in the low-mass WIMP region. To calculate the expected WIMP rate in the detectors, we follow the commonly used analysis framework for WIMP direct detection [27,28]. We consider only the spin-independent (SI) contribution since the spin-dependent contribution is comparatively reduced in $\mathrm{TeO}_{2}$; the main isotopes with non-zero nuclear spin are ${ }^{125} \mathrm{Te}$ and ${ }^{123} \mathrm{Te}$, with isotopic abundance of $7.1 \%$ and $0.9 \%$, respectively. We assume coherent isospin-invariant coupling and the Helm model [29] for the nuclear form factors. Under these assumptions, the generic WIMP is completely determined by its mass $m_{\mathrm{W}}$ and SI WIMP-nucleon cross section $\sigma_{\mathrm{SI}}$. For the velocity distribution of dark matter, we use the standard halo model (SHM) [30] commonly adopted for comparisons of direct detection experiments. Consequently, the annually modulating WIMP recoil rate due to the motion of the Earth around the Sun can be approximated using a constant term $S_{0}$ plus a cosine-modulated term $S_{m}$, as given by

$\frac{d R_{W}}{d E}(E, t)=S_{0}(E)+S_{m}(E) \cos \left[\omega\left(t-t_{0}\right)\right]$

where $\omega=2 \pi /$ year and $t_{0}$ is around June 1 .
To obtain the sensitivity to annual modulation of WIMPs, we scan over the WIMP parameter space $\left(m_{\mathrm{W}}, \sigma_{\mathrm{SI}}\right)$ looking for the region at which a WIMP interaction would produce an annual modulation in the detection rate over the measured background at a certain confidence level (C.L.). For each $\left(m_{\mathrm{W}}, \sigma_{\mathrm{SI}}\right)$ we generate 100 toy $\mathrm{MC}$ simulations and for each MC spectrum, we perform a maximum likelihood (ML) analysis for both the annual-modulation (AM) and the absence of modulation (null) hypotheses. We quote the significance of the modulation as the log-likelihood ratio of the best fits $\chi^{2}=2 \log \left(\mathcal{L}_{\mathrm{AM}} / \mathcal{L}_{\text {null }}\right)$. The likelihood $\mathcal{L}_{\mathrm{AM}}$ is calculated using the probability density function (PDF)

$$
\begin{aligned}
\phi= & \frac{d R_{W}}{d E}\left(E, t ; m_{W}, \sigma_{\mathrm{SI}}\right) M_{\mathrm{det}} \varepsilon_{\mathrm{BoDs}}(E, t) \\
& +\phi_{\mathrm{b}}\left(E ; a_{i}\right) \varepsilon_{\mathrm{BoDs}}(E, t)
\end{aligned}
$$

where $M_{\text {det }}$ is the target mass, $\varepsilon_{\mathrm{BoDs}}(E, t)$ is the BoDsdependent detection efficiency, and $\phi_{\mathrm{b}}$ is the background PDF, which we model with a Chebychev polynomial with coefficients $a_{i}$ and for which we do not consider any temporal dependence. The likelihood $\mathcal{L}_{\text {null }}$ is calculated from $\phi_{\mathrm{b}}$ alone.

We choose the ROI to perform the analysis as $10-28 \mathrm{keV}$, which excludes the peak-like structures above $30 \mathrm{keV}$ shown in Fig. 5. Given that the differential rate of WIMPs quasiexponentially falls as a function of energy, most of the signal is contained at the low energy and the expected contribution to the WIMP sensitivity from 30 to $60 \mathrm{keV}$ is negligible compared to that from 10 to $28 \mathrm{keV}$. We consider a target mass of $742 \mathrm{~kg}$ and the scheduled 5 years of data-taking with $75 \%$ duty cycle, accounting for the calibration time. Based on the CUORE-0 energy threshold, we use $10 \mathrm{keV}$ but we also show the sensitivity that could be attained under the more optimistic hypothesis that we reach a $3 \mathrm{keV}$ threshold as demonstrated in the CCVR experiment with a linear extrapolation of the CUORE-0 background to lower energies.

Figure 6 shows CUORE sensitivity requiring a 90\% C.L. in $90 \%$ of the toy-MC experiments. The results are consistent with those obtained with a pure statistical calculation following [36]. This figure assumes a WIMP local density $\rho_{\chi}=0.3 \mathrm{GeV} / c^{2}$, local circular velocity $v_{0}=220 \mathrm{~km} / \mathrm{s}$, galactic escape velocity $v_{\mathrm{esc}}=650 \mathrm{~km} / \mathrm{s}$, and orbital velocity of the Earth around the Sun $v_{\text {orb }}=29.8 \mathrm{~km} / \mathrm{s}$. Uncertainty on the energy scale dominated by the nuclear quenching factor is taken into account. For comparison, we also show the $5 \sigma, 3 \sigma$ and $90 \%$ C.L. regions resulting from a ML analysis reported in [31] on the DAMA/LIBRA annual modulation positive result $[37,38]$ using the same parameters for the SHM. Thanks to the $741 \mathrm{~kg}$ of target mass of CUORE, we expect to achieve the sensitivity required to fully explore the parameter region implied by the DAMA/LIBRA posi- 
tive annual modulation signal with 5 years of data-taking. Other recent experimental results from CRESST-II, CDMS Lite, XMASS and LUX [32-35] are also shown. The results from CRESST-II, CDMS, and LUX were obtained using $v_{\text {esc }}=544 \mathrm{~km} / \mathrm{s}$. The impact of using $v_{\mathrm{esc}}=544 \mathrm{~km} / \mathrm{s}$ instead of $v_{\text {esc }}=650 \mathrm{~km} / \mathrm{s}$ for CUORE sensitivity is found to be less than $10^{-5} \mathrm{pb}$ at $6 \mathrm{GeV}$. Also for the other experiments only a minor impact of the escape velocity on the exclusion limit is expected.

\section{Summary}

We have presented the analysis techniques developed for low energy rare event searches with CUORE and their validation using the data acquired with the CUORE- 0 experiment. We have optimized the software trigger developed in previous CUORE prototypes, removing an intrinsic dead time that prevented the algorithm from reaching $100 \%$ efficiency, and designed a protocol to periodically monitor the efficiency by injecting low energy reference pulses at the end of every dataset. With the new trigger, we have reduced the CUORE-0 trigger thresholds from several tens of $\mathrm{keV}$ to values between 4 and $12 \mathrm{keV}$.

We have also demonstrated that a pulse shape analysis can efficiently select legitimate physics events in $\mathrm{TeO}_{2}$ bolometers against spurious ones at energies below $100 \mathrm{keV}$. In addition, we have developed a technique, scalable to an experiment with one thousand bolometers, to independently establish the analysis threshold of each bolometer in each dataset. In CUORE-0 the analysis thresholds range between 8 and $35 \mathrm{keV}$. Using characteristic X-rays from Te, we have found the energy scale shift to be $0.18 \pm 0.13 \mathrm{keV}$ upward at $\sim 27 \mathrm{keV}$.

After the data and event selection, the CUORE-0 background rate ranges from 1.7 counts/(kg keV day) at $10 \mathrm{keV}$ to 0.05 counts / ( $\mathrm{kg} \mathrm{keV}$ day) at $50 \mathrm{keV}$, two times less than that attained with the best Cuoricino bolometers. Nevertheless, the low energy spectrum requires further investigation including the explanation of the peak-like structures between 30 and $40 \mathrm{keV}$. We use the nuclear quenching factors of $\mathrm{TeO}_{2}$ obtained by tagging the recoiling daughter nuclei of $\alpha$ decays in the CUORE-0 data to estimate the uncertainty of the WIMP energy scale. We incorporate it to report the CUORE sensitivity to WIMP annual modulation.

CUORE will search for low energy rare events such as solar axions, WIMP dark matter in the galactic halo, or coherent scattering of galactic supernova neutrinos using the analysis techniques presented in this paper. In particular, we expect to reach a sensitivity to annual modulation of WIMPs sufficient to fully explore the parameter region indicated by the positive annual modulation signal of the DAMA/LIBRA experiment with 5 years of CUORE data-taking.
Acknowledgements The CUORE Collaboration thanks the directors and staff of the Laboratori Nazionali del Gran Sasso and our technical staff for their valuable contribution to building and operating the detector. This work was supported by the Istituto Nazionale di Fisica Nucleare (INFN); the National Science Foundation under Grant Nos. NSFPHY-0605119, NSF-PHY-0500337, NSF-PHY-0855314, NSF-PHY0902171, NSF-PHY-0969852, NSF-PHY-1307204, and NSF-PHY1404205; the Alfred P. Sloan Foundation; the University of Wisconsin Foundation; and Yale University. This material is also based upon work supported by the US Department of Energy (DOE) Office of Science under Contract Nos. DE-AC02-05CH11231 and DE-AC5207NA27344; and by the DOE Office of Science, Office of Nuclear Physics under Contract Nos. DE-FG02-08ER41551 and DEFG0300ER41138. This research used resources of the National Energy Research Scientific Computing Center (NERSC).

Open Access This article is distributed under the terms of the Creative Commons Attribution 4.0 International License (http://creativecomm ons.org/licenses/by/4.0/), which permits unrestricted use, distribution, and reproduction in any medium, provided you give appropriate credit to the original author(s) and the source, provide a link to the Creative Commons license, and indicate if changes were made.

Funded by SCOAP S $^{3}$

\section{References}

1. C. Arnaboldi et al., Nucl. Instrum. Meth. A 518, 775 (2004). https:// doi.org/10.1016/j.nima.2003.07.067

2. D. Artusa et al., Adv. High Energy Phys. 2015, 879871 (2015). https://doi.org/10.1155/2015/879871

3. M. Redshaw, B.J. Mount, E.G. Myers, F.T. Avignone III, Phys. Rev. Lett. 102(21), 212502 (2009)

4. N.D. Scielzo et al., Phys. Rev. C 80, 025501 (2009)

5. S. Rahaman et al., Phys. Lett. B 703(4), 412 (2011)

6. C. Alduino et al., Eur. Phys. J. C 77(1), 13 (2017). https://doi.org/ 10.1140/epjc/s10052-016-4498-6

7. E. Andreotti et al., Astropart. Phys. 34, 822 (2011)

8. K. Alfonso et al., Phys. Rev. Lett. 115(10), 102502 (2015). https:// doi.org/10.1103/PhysRevLett.115.102502

9. C. Arnaboldi et al., Astropart. Phys. 20, 91 (2003). https://doi.org/ 10.1016/S0927-6505(03)00180-4

10. M.W. Goodman, E. Witten, Phys. Rev. D 31, 3059 (1985). https:// doi.org/10.1103/PhysRevD.31.3059

11. A.K. Drukier, K. Freese, D.N. Spergel, Phys. Rev. D 33, 3495 (1986). https://doi.org/10.1103/PhysRevD.33.3495

12. K. Freese, J.A. Frieman, A. Gould, Phys. Rev. D 37, 3388 (1988). https://doi.org/10.1103/PhysRevD.37.3388

13. F. Alessandria et al., JCAP 1305, 007 (2013). https://doi.org/10. 1088/1475-7516/2013/05/007

14. S. Di Domizio, F. Orio, M. Vignati, JINST 6, P02007 (2011). https://doi.org/10.1088/1748-0221/6/02/P02007

15. F. Alessandria et al., Astropart. Phys. 35, 839 (2012)

16. F. Alessandria et al., JCAP 1301, 038 (2013). https://doi.org/10. 1088/1475-7516/2013/01/038

17. C. Alduino et al., JINST 11(07), P07009 (2016). https://doi.org/ 10.1088/1748-0221/11/07/P07009

18. E.E. Haller, N.P. Palaio, M. Rodder, W.L. Hansen, E. Kreysa, NTD germanium: a novel material for low temperature bolometers (Springer US, Boston, MA, 1984), pp. 21-36. https://doi.org/ 10.1007/978-1-4613-2695-3_2

19. E. Andreotti, C. Brofferio, L. Foggetta, A. Giuliani, B. Margesin, C. Nones, M. Pedretti, C. Rusconi, C. Salvioni, M. Tenconi, Nucl. Instrum. Meth. A664, 161 (2012). https://doi.org/10.1016/j.nima. 2011.10.065 
20. C. Alduino et al., Phys. Rev. C 93, 045503 (2016). https://doi.org/ 10.1103/PhysRevC.93.045503

21. E. Gatti, P.F. Manfredi, Riv. Nuovo Cim. 9N1, 1 (1986). https:// doi.org/10.1007/BF02822156

22. G. Piperno, J. Phys. Conf. Ser. 718(4), 042045 (2016). https://doi. org/10.1088/1742-6596/718/4/042045

23. S.Y.F. Chu, L.P. Ekstrom, R.B. Firestone. The lund/lbnl nuclear data search. http://nucleardata.nuclear.lu.se/toi/xray.asp?act=list\& $\mathrm{el}=\mathrm{Te}$

24. S. Agostinelli et al., Nucl. Instr. Meth. A 506, 250 (2003). https:// doi.org/10.1016/S0168-9002(03)01368-8

25. C. Alduino et al., Eur. Phys. J. C 77(8), 543 (2017). https://doi.org/ 10.1140/epjc/s10052-017-5080-6

26. A. Alessandrello et al., Phys. Lett. B 408, 465 (1997). https://doi. org/10.1016/S0370-2693(97)00765-X

27. P.F. Smith, J.D. Lewin, Phys. Rept. 187, 203 (1990). https://doi. org/10.1016/0370-1573(90)90081-C

28. J.D. Lewin, P.F. Smith, Astropart. Phys. 6, 87 (1996). https://doi. org/10.1016/S0927-6505(96)00047-3

29. R.H. Helm, Phys. Rev. 104, 1466 (1956). https://doi.org/10.1103/ PhysRev.104.1466
30. K. Freese, M. Lisanti, C. Savage, Rev. Mod. Phys. 85, 1561 (2013). https://doi.org/10.1103/RevModPhys.85.1561

31. C. Savage, G. Gelmini, P. Gondolo, K. Freese, JCAP 0904, 010 (2009). https://doi.org/10.1088/1475-7516/2009/04/010

32. G. Angloher et al., Eur. Phys. J. C 76(1), 25 (2016). https://doi.org/ 10.1140/epjc/s10052-016-3877-3

33. R. Agnese et al., Phys. Rev. Lett. 116(7), 071301 (2016). https:// doi.org/10.1103/PhysRevLett.116.071301

34. K. Abe et al., Phys. Lett. B 759, 272 (2016). https://doi.org/10. 1016/j.physletb.2016.05.081

35. D.S. Akerib et al., Phys. Rev. Lett. 112, 091303 (2014). https://doi. org/10.1103/PhysRevLett.112.091303

36. S. Cebrian et al., Astropart. Phys. 14, 339 (2001). https://doi.org/ 10.1016/S0927-6505(00)00124-9

37. R. Bernabei et al., Eur. Phys. J. C 56, 333 (2008). https://doi.org/ 10.1140/epjc/s10052-008-0662-y

38. R. Bernabei et al., Eur. Phys. J. C 73, 2648 (2013). https://doi.org/ 10.1140/epjc/s10052-013-2648-7 